\title{
High-Contact-First Immunization Strategy on Complex Networks
}

\author{
Fuzhong Nian ${ }^{\mathrm{a}^{*}}$, Longjing Wang ${ }^{\mathrm{b}}$, Tong Liu and Ke Wang \\ School of Computer \& Communication, Lanzhou University of Technology, China \\ agdnfz@lut.cn, bwanglongjing.china@gmail.com
}

Keywords: Complex networks; Immunization; High-contact; Spreading

\begin{abstract}
Different from traditional immunization strategies, which suppose that the contact frequency of all nodes is same, in this paper, a new immunization strategy was established based on the principle of 'High-Contact-First'. The different link with different weight describes the relationship between different two nodes. The bigger weight means the higher contact. The higher contact means the higher risk which the two nodes be infected each other. Thus, to the purpose of immunization, the link with higher contact should be cut down first. The theory analysis and the simulations indicate that the proposed immunization strategy is effective, and it is also economic and feasible.
\end{abstract}

\section{Introduction}

Since the human contact patterns and the topology of Internet can be regarded as complex networks, the epidemic spreading on complex networks, include the spreading of computer viruses and human diseases has been widely investigated[1-6]. In particular, the spreading of human disease in realistic systems and theoretical models has attracted a great deal of attention[7-12]. And a vast amount of work has been done. The typical epidemic models include SI, SIS, SIR and SIRS etc. In the SI model[13] and SIS model[14] individuals can only exist in two discrete states: susceptible or infected. While, in the SIR model[15] and SIRS model[11], individual scan be one of the three states: susceptible, infected, and recovered. In SIRS model, an infected node can be recovered again, and after a period, it would be a susceptible node, because the losing of immunity.

How the infectious disease propagates in population, and how to control the speed of spread of the epidemic? These are the important questions in the spread of epidemic on complex networks. Recently, many methods were proposed to solve the problem[1,16-19].

Generally, the most common immunization strategies include random immunization, targeted immunization and acquaintance immunization. Random immunization[20] is also called uniform immunization which was put forward by Anderson and May in 1992. The vaccinated nodes are selected randomly at a certain proportion. The results showed that in order to obtain a better immune effect, almost all individuals need to be vaccinated. Then Pastor-Satorras and Vespignani proposed the targeted immunization[21] in 2001. In targeted immunization, the nodes with high degrees were vaccinated firstly. The targeted immunization has a better immune effect, but it needs to know the topological structure of the whole network. In response to this issue, Cohen designs an efficient immunization strategy named "acquaintance immunization"[22]. In this scheme, the "acquaintances" of some nodes are randomly selected and be vaccinated with certain probability. It is evident that the nodes with large degree have more chance to be vaccinated.

All the previous studies, there is a hypothesis which the frequency of contact between any two individuals is same, and the immune probability of each node is same too. However, the contact frequency of different two individuals is different in general. In many nature or social networks, individuals tend to form groups according to the different characteristics, such as different jobs, different families and so forth. The connections within each group are dense, while connections between groups are sparse. For example, in social networks, family is a group. Within a family group, each member is directly connected each other, but connections among different families are relatively rare. Based on this situation, in some degree, structure determines the characteristics and 
function of networks. Thus, how to describe the contact frequency between individuals and how to choose the effective immunization strategy is an important work.

In this paper, the weights of links between all nodes in the networks are considered. And the different weights describe the different contact frequency between the relative nodes. According to the weighted networks, a "high-contact-first" immunization strategy was proposed. In high-contact-first immunization, the nodes which are connected to the infected nodes and with high contact frequency should be vaccinated firstly. To verify the validity of the immunization, the theoretical proof and computer simulations were done on different networks with SIRS epidemic model. The results indicate that the proposed immunization strategies are effective, and it is also economic and feasible.

The rest of the paper is organized as follows: the high-contact immunity in SIRS model in small-world networks of the epidemic model and the simulation results are presented in Sec. I. The high-contact immunity in SIRS model in scale-free networks of the epidemic model and the simulation results are presented in Sec. II and the conclusion is presented in Sec. III.

\section{High-Contact-First Immunization in Small-Word Networks}

The characteristic of WS small-world model is the connectivity distribution can be approximately represented by the Poisson distribution. The distribution has a peak in the average value $\langle\mathrm{k}\rangle$, then rapid degradation exponentially, which means that when $\mathrm{k}\rangle\langle\mathrm{k}\rangle$, it almost not exists a node which degree is K. So the networks also known as the Homogeneous network. Suppose there are N nodes in the WS small-world network, each node is symmetrical connected to the nearest $\mathrm{K}$ nodes with probability $\mathrm{p}$ randomly re-connected to the each edge in the original network, and then the average degree of the network is $\langle\mathrm{k}\rangle=2 \mathrm{~K}$. In the real social network, the interpersonal contact frequency is influenced by many independent factors, so the interpersonal contact frequency is meet the normal distribution. It is described as:

$$
C \sim N\left(\mu, v^{2}\right)
$$

Here, $C$ represents the weight of edge. $\mu$ is the average value of the weight of all edges. $v$ is the variance of all weight. The high-contact-first immunization strategy was described as: the nodes connected to the initial infection nodes with big weight are vaccinated first. It means the biggest potential threaten should be eliminated first in the networks. Thus, the immune probability is proportional to the weights. It is evident that the high-contact-first immunization is a dynamic strategy.

Let $\delta$ represents the probability of a susceptible individual were vaccinated. Then,

$$
\delta \sim a c_{i j}
$$

Where, $c_{i j}$ is the weight between the healthy individual $j$ and the infected node $i$. $a$ is constant coefficient. For the convenience of calculation, let $a$ equal to 1 .

The dynamic equations of the SIRS propagation model on WS small-world network[16,19,23] are described as follows:

$$
\begin{aligned}
& \frac{d S(t)}{d t}=-\lambda<k>S(t) I(t)+\sigma R(t) \\
& \frac{d I(t)}{d t}=\lambda<k>S(t) I(t)-I(t) \\
& \frac{d R(t)}{d t}=I(t)-\sigma R(t)
\end{aligned}
$$

Where, $S(t), I(t)$, and $R(t)$, respectively, denote average density of susceptible individuals, infected individuals and removed individuals. The infection transmission is defined by the rate $\beta$ at which each susceptible individual acquires the infection from an infected neighbor during one time step. The rate constant of recovery for infected individuals is denoted by $\Upsilon$. Then the model's 
effective spreading rate is $\lambda=\beta / \Upsilon . \sigma$ represents that the recovered node loses its immunity with rate, $\langle k\rangle$ is average degree of the networks. High-risk immunization is regard the susceptible individuals with infected neighbors as high-risk groups, and by immunizing the high-risk groups with a certain probability[16,19] to controls the spread of the disease When the node is vaccinated, it means that a potential virus transmission channel is eliminated. However, in many cases, the influence of every edge is not equal because of the different of the contact frequency. For example, the contact frequency of family members is far high from the others generally. So, the vaccination should be treated differently. Certainly, the susceptible individual with high contact frequency to the infected should be should be vaccinated firstly. It is called as high-contact-first immunization.

The individuals were vaccinated according to the order of contact frequency between healthy nodes and infected nodes in high-contact-first immunization strategy. Evidently, it is more easily to eliminate the transmission channel with the biggest risk. At the same time, the individuals with low contact frequency were ignored selectively. Compared with the high-risk immunization strategy, high-contact-first immunization strategy has lower cost.

Based on Eq.(3), we can get the dynamic equations of high-contact-first immunization:

$$
\begin{aligned}
& \frac{d S(t)}{d t}=-\lambda k\left(1-\sum_{x=1, y=1}^{N} c_{x y} \Omega / \sigma\right) \sum_{i=1, j=1}^{N} c_{i j} S(t) I(t)+\sigma R(t) \\
& \frac{d I(t)}{d t}=\lambda k\left(1-\sum_{x=1, y=1}^{N} c_{x y} \Omega / \sigma\right) \sum_{i=1, j=1}^{N} c_{i j} S(t) I(t)-I(t) \\
& \frac{d R(t)}{d t}=I(t)-\sigma R(t)
\end{aligned}
$$

Here, $c_{x y}$ represents the vaccinated probability of a healthy node $y$ who connected to the infected node $x \quad\left(c_{x y}\right.$ is the weight between node $\mathrm{x}$ and node $\left.\mathrm{y}\right) . c_{i j}$ represents contact frequency between node $i$ and node $j . S(t), I(t)$, and $R(t)$ respectively represent different density about healthy nodes, infected nodes and recovered nodes. $\Omega$ is the probability that any given node is a neighbor of some specific node. Hence, the effective transmission rate decreases by rate $1-\sum_{x=1, y=1}^{N} c_{x y} \Omega / \sigma$, and it means that $\lambda \rightarrow \lambda\left(1-\sum_{x=1, y=1}^{N} c_{x y} \Omega / \sigma\right)$.

Also, $S(t), I(t)$ and $R(t)$ obey the normalization condition:

$$
S(t)+I(t)+R(t)=1
$$

When the network tends to the steady state of no infectious diseases spread on networks are:

$$
\begin{aligned}
& \frac{d S(t)}{t}=0 \\
& \frac{d I(t)}{t}=0 \\
& \frac{d R(t)}{t}=0 \\
& I(t)=0
\end{aligned}
$$

This means that all states do not change, and there is no disease on population now. Then, from the first equation of Eq. (4) and Eq.(6), we obtain:

$$
-\lambda k\left(1-\sum_{x=1, y=1}^{N} c_{x y} \Omega / \sigma\right) \sum_{i=1, j=1}^{N} c_{i j} S(t) I(k)+\sigma R(t)=0
$$

From the second equation of Eq. (4) and Eq.(6), we know: 


$$
S(t)=\frac{1}{-\lambda k\left(1-\sum_{x=1, y=1}^{N} c_{x y} \Omega / \sigma\right) \bar{c}}
$$

$c$ is average of all weights. At the same time, Substitute Eq. (8) into Eq. (7):

$$
-I+\sigma\left[1-I-\frac{1}{\lambda\left(1-\sum_{x=1, y=1}^{N} c_{x y} \Omega / \sigma\right)^{-}}\right]=0
$$

Because $I(t)=0$, we can get:

$$
\frac{1}{\lambda\left(1-\sum_{x=1, y=1}^{N} c_{x y} \Omega / \sigma\right)<k>\bar{c}}=1
$$

At this point, a non-zero threshold can be obtained:

$$
\lambda_{c}=\frac{1}{\left(1-\sum_{x=1, y=1}^{N} c_{x y} \Omega / \sigma\right)<k>\bar{c}}
$$

When contact frequency among different nodes is same and the weight is $1, \lambda_{c}$ satisfies that $\lambda_{c}=\frac{1}{<k>}$. It is totally consist with the condition in the SIS propagation model[24]. And, if the weight is not equal to $1\left(0<\mathrm{c}_{\mathrm{xy}}<1\right)$, obviously, $\lambda_{C}>\frac{1}{<k>}$. So, is the high-contact-first immunization strategy effective? We will provide answers by using simulation experiments.

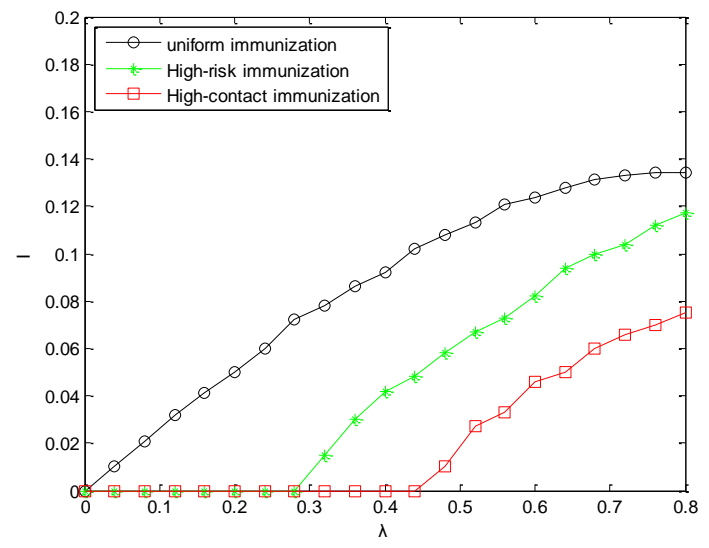

Figure 1. Three different immunization strategies in the small-world network ( $N=2000$, randomly re-connected probability $\mathrm{p}=0.2,\langle\mathrm{k}\rangle=4$, Infection density varies with propagation probability)

All the simulations (Fig.1-Fig.4) are computed averaging over 30 different starting configurations, performed on 20 different realizations of the network. Here, $10 \%$ of the nodes in the network are chosen randomly as initial infected nodes in a network. We choose 0.1 as the probability that the node loses immunity. For simplicity, the "high-contact-first" was replaced by "high-contact" in all simulation figures.

In Fig. 1, the random immunization and high-risk immunization ignore the contact frequency between the nodes and $\delta=0.3$. While high-contact-first immunization, the contact frequency satisfy $C \sim N(1 / 2,1)$. Obviously, we can see, in the Fig.1 the effect of high-risk immunization is better 
than random immunization, and when $\delta=0.3$, the effect is slightly worse than the high-contact-first immunization.

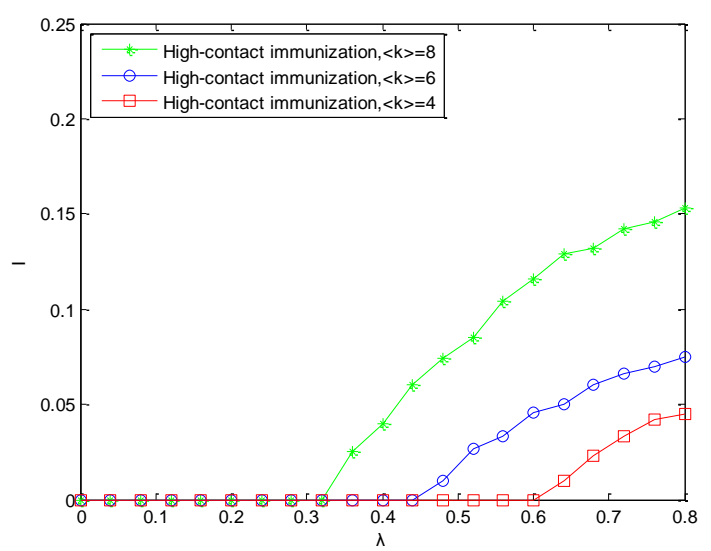

Figure 2. Comparison of high-contact-first immunization indifferent networks with different average degree $(\mathrm{N}=2000, \mathrm{p}=0.2$, Infection density varies with propagation probability)

In Fig.2, it shows the comparison of high-contact-first immunization when $\langle k\rangle$ is variety. We can get the conclusion that: when $\langle k\rangle$ gets smaller, the effect become better. The situation also satisfies the fact in the real world. A bigger $\langle k>$ means there are more edges in the networks, it also indicates more transmission channels for infectious diseases.

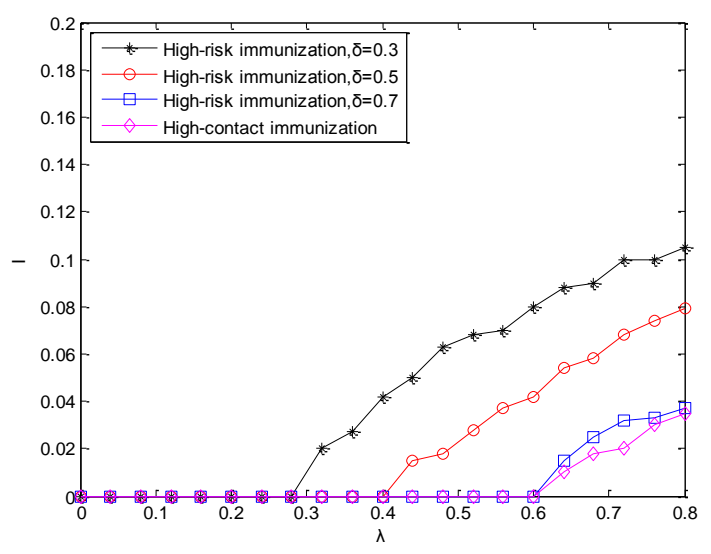

Figure 3. Contrast between high-risk immunization and high-contact-first immunization $(\mathrm{N}=2000$, $\mathrm{p}=0.2,<\mathrm{k}>=4$, the variation of infected density I changes with the function of $\lambda$.)

In Fig.3, by comparing two kinds of immunization strategies, when $\delta$ gets bigger $(\delta=0.7)$, the effect of high-risk immunization is approximately equal to high-contact-first immunization. In the past study, the probability that nodes get vaccinated was same, and ignored their contact frequency. While, for high-contact-first immunization, its purpose is to vaccinate the nodes connected to infected nodes and the contact frequency is bigger.

In homogeneous networks, each node is approximately equal to the average degree. When the density of initial infected nodes is consistent, the result is often quite similar. Different from homogeneous networks, in heterogeneous networks, a few nodes tend to have larger degree. Once these nodes get infected, it means more risks for healthy nodes.

\section{High-Contact-First Immunization in Scale-Free Networks}

In BA network, connection degree distribution function has the power-law form and the nodes' degree without significantly characteristic length, so called scale-free networks. Barabási and Albert proposed the BA scale-free model based on the characteristic of growth and preferential attachment. Here, BA stands for Barabási-Albert. We construct a BA scale-free network composed of N nodes 
with degree distribution $\mathrm{P}(\mathrm{k}) \sim \mathrm{k}^{-3}$ and average degree is $\langle\mathrm{k}\rangle=2 \mathrm{~m}$. For the BA scale-free networks[25], due to the scale-free property, nodes with high degrees play an important role in networks. As long as propagation rate is greater than zero, the virus can spread and finally maintained at a stable state. This reflects the vulnerability of the scale-free network against the virus. So, finding a suitable immunization strategy is very important. Does high-contact-first immunization strategy in the BA scale-free network perform well? Now, we will show the performance through the theoretical analysis and simulation experiment.

Because the BA network is heterogeneous, so, the SIRS model which modeled base on mean-field theory should be modified. Let $S_{k}(t)$ is the density of susceptible nodes, $I_{k}(t)$ is the density of infected nodes and $R_{k}(t)$ is the density of recovered nodes with a given degree $k$. The propagation model we adopted is SIRS built on BA scale-free networks. The dynamic equations as follows:

$$
\begin{aligned}
& \frac{d S_{k}(t)}{d t}=-\lambda k\left(1-\sum_{x=1, y=1}^{N} c_{s t} \Omega / \sigma\right) \Theta(t) \sum_{i=1, j=1}^{N} c_{i j} S_{k}(t)+\sigma R_{k}(t) \\
& \frac{d I_{k}(t)}{d t}=\lambda k\left(1-\sum_{x=1, y=1}^{N} c_{s t} \Omega / \sigma\right) \Theta(t) \sum_{i=1, j=1}^{N} c_{i j} S_{k}(t)-I_{k}(t) \\
& \frac{d R_{k}(t)}{d t}=I_{k}(t)-\sigma R_{k}(t)
\end{aligned}
$$

The first term on the rhs of the first equation of Eq. (12) considers the average density of newly infected nodes generated by each infected node. It is proportional to the effective infection spreading rate $\lambda$, the number of connections $k$ (degree), and the probability that a given link points to a healthy node $S_{k}(t)$. The probability $\Omega$ is represent a given node is the neighbor of a specific node. The rest terms in Eq. (12) are similar to above.

The probability that a link points to a node with $s$ links is proportional to $s P(s)$. In other words, a randomly chosen link is more likely to be connected to an infected node with high connectivity, yielding[26]:

$$
\Theta(t)=\frac{\sum_{k} k P(k) I_{k}(t)}{\sum_{s} s P(s)}=\frac{1}{<k>} \sum_{k} k P(k) I_{k}(t)
$$

Also, $S_{k}(t), I_{k}(t)$ and $R_{k}(t)$ obey the normalization condition for each $k$ :

$$
S_{k}(t)+I_{k}(t)+R_{k}(t)=1
$$

Consider the non-epidemic stationary condition:

$$
\begin{aligned}
& \frac{d S_{k}(t)}{d t}=0 \\
& \frac{d I_{k}(t)}{d t}=0 \\
& \frac{d R_{k}(t)}{d t}=0
\end{aligned}
$$

Then, from the first equation of Eq. (12), we obtain

$$
-\lambda k\left(1-\sum_{x=1, y=1}^{N} c_{s t} \Omega / \sigma\right) \Theta(t) \sum_{i=1, j=1}^{N} c_{i j} S_{k}(t)+\sigma R_{k}(t)=0
$$

Combine the above equation with Eq. (14), we obtain 


$$
S_{k}(t)=\frac{\sigma-\sigma I_{k}(t)}{-\lambda k\left(1-\sum_{x=1, y=1}^{N} c_{s t} \Omega / \sigma\right) \Theta(t) \bar{c}+\sigma}
$$

$\bar{c}$ is the average of all weights. From the second equation of Eq. (12)

$$
S_{k}(t)=I_{k}(t) / \lambda k\left(1-\sum_{x=1, y=1}^{N} c_{s t} \Omega / \sigma\right) \Theta(t) \bar{c}
$$

Substitute Eq. (18) into Eq. (17)

$$
I_{k}(t)=\frac{\sigma \lambda k\left(1-\sum_{x=1, y=1}^{N} c_{s t} \Omega / \sigma\right) \Theta(t) \bar{c}}{(1+\sigma) \lambda k\left(1-\sum_{x=1, y=1}^{N} c_{s t} \Omega / \sigma\right) \Theta(t) \bar{c}+\sigma}
$$

The solution $I_{k}(t)=0$ and $\Theta(t)=0$ always satisfy the Eq.(19). A nonzero stationary prevalence $\left(I_{k}(t) \neq 0\right)$ is obtained when the lhs and the rhs of Eq.(19), expressed as function $F(\Theta)$, and interest is in the interval $0<\Theta \leq 1$; this has a nontrivial solution. It is easy to deduce that this requires that the inequality $\left.\frac{d F(\Theta)}{d \Theta}\right|_{\Theta=0} \geq 1$.

To solve the equation $\frac{d F(\Theta)}{d \Theta}$, we can get

$$
\frac{d F(\Theta)}{d \Theta}=\frac{\lambda}{<k>} \sum_{k} k P(k)\left(1-\sum_{x=1, y=1}^{N} c_{s t} \Omega / \sigma\right) \bar{c} \geq 1
$$

$\Omega$ is the probability of any given node (individual) is a neighbor of some specific nodes. Therefore:

$$
\Omega=k P(k) / N<k>
$$

As for BA scale-free network

$$
P(k)=2 m^{2} / k^{3}
$$

Substitute Eq. (22) and Eq. (21) into the right side of inequality (20), we obtain

$$
\frac{\lambda}{<k>}\left[<k^{2}>-\frac{2 m^{2} \sum_{x=1, y=1}^{N} c_{s t}}{N<k>\sigma}\right] \bar{c} \geq 1
$$

From above equation, the critical epidemic threshold $\lambda_{c}$, that is given by

$$
\lambda_{c}=\frac{N<k>\sigma}{\bar{c}\left(N<k^{2}>\sigma-2 m^{2} \sum_{x=1, y=1}^{N} c_{s t}\right)}
$$

When $\sigma=1, \bar{c}=1$ and $c_{s t}=1(1 \leq s, t \leq N)$, we can get $\lambda_{c}=\frac{<k>}{<k^{2}>}$. This is consistent with the May's reference[13].

Here 


$$
\begin{aligned}
& <k>=\sum_{k} k P(k)=\sum_{k} 2 m^{2} / k^{2}=2 m^{2} \sum_{k} \frac{1}{k^{2}} \\
& <k^{2}>=\sum_{k} k^{2} P(k)=\sum_{k} k^{2} 2 m^{2} / k^{3}=2 m^{2} \sum_{k} \frac{1}{k}
\end{aligned}
$$

Substitute Eqs.(25) into Eq. (24)

$$
\lambda_{c}=\frac{2 N m^{2}\left(\sum_{k} \frac{1}{k^{2}}\right)^{2} \sigma}{\left[2 N m^{2}\left(\sum_{k} \frac{1}{k^{2}}\right) \sum_{k} \frac{1}{k} \sigma-\sum_{x=1, y=1}^{N} c_{s t}\right] \bar{c}}=\left\{\frac{\sum_{k} \frac{1}{k}}{\sum_{k} \frac{1}{k^{2}}}-\frac{\delta}{2 N m^{2}\left(\sum_{k} \frac{1}{k^{2}}\right)^{2} \sum_{x=1, y=1}^{N} c_{s t}}\right\} \bar{c}
$$

In the continuous $k$ approximation, calculate the approximation of $\sum_{k} \frac{1}{k}$ and $\sum_{k} \frac{1}{k^{2}}$

$$
\begin{aligned}
& \sum_{k} \frac{1}{k} \rightarrow \int_{m}^{M} \frac{1}{k} d k=\ln \frac{M}{m} \\
& \sum_{k} \frac{1}{k^{2}} \rightarrow \int_{m}^{M} \frac{1}{k^{2}} d k=\frac{1}{m}-\frac{1}{M}=\frac{M-m}{m M}
\end{aligned}
$$

Here, $M$ is the maximum degree. Substitute Eqs. (27) into Eq. (26):

$$
\begin{aligned}
& \frac{1}{\lambda_{c}} \approx\left\{\frac{\ln \frac{M}{m}}{\frac{M-m}{m M}}-\frac{\sum_{x=1, y=1}^{N} c_{s t}}{2 N m^{2}\left(\frac{M-m}{m M}\right)^{2} \sigma}\right\} \bar{c} \\
& =\left\{\frac{m M \ln \frac{M}{m}}{M-m}-\frac{M^{2} \sum_{x=1, y=1}^{N} c_{s t}}{2 N(M-m)^{2} \sigma}\right\} \bar{c}
\end{aligned}
$$

When $M$ is big enough (when $m$ is constant, $M$ increases with $N \quad$ ), $M-m \approx M$ thus

$$
\frac{1}{\lambda_{c}} \approx\left\{m \ln \frac{M}{m}-\frac{M \sum_{x=1, y=1}^{N} c_{s t}}{2 N \sigma}\right\} \bar{c}
$$

From Eq. (29), we can see $\lambda_{c}$ increases with $\sigma$ increasing; $\lambda_{c}$ increases with $m(k=2 m)$ decreasing; $\lambda_{c}$ decreases with $N$ increasing. Performance of these three points in real life: First, longer lasting immunity raises the threshold infection spreading rate for epidemic outbreak to occur, in this section, we use 0.1 as the probability that nodes lose its immunity; Second, when one node in the network has more edges, it undertake more risk; Third, when the size of the population increases, the outbreak of infectious diseases get easier. In this paper, we assume $\bar{c}=0.5$.

To verify the above analysis, some simulations were implemented on computer. The results indicate that the analysis is correct. The network was selected as scale-free network with $N=2000$, average degree $\langle k\rangle=4$, and the initial node $\mathrm{m}=5$. The $0.1 * N$ infected nodes were selected randomly from the network. Suppose the chance of be infected by infected neighbor is equal to all nodes, and the probability of infection is the same. Simulations were computed averaging over 30 different starting configurations, performed on 20 different realizations of the network. 


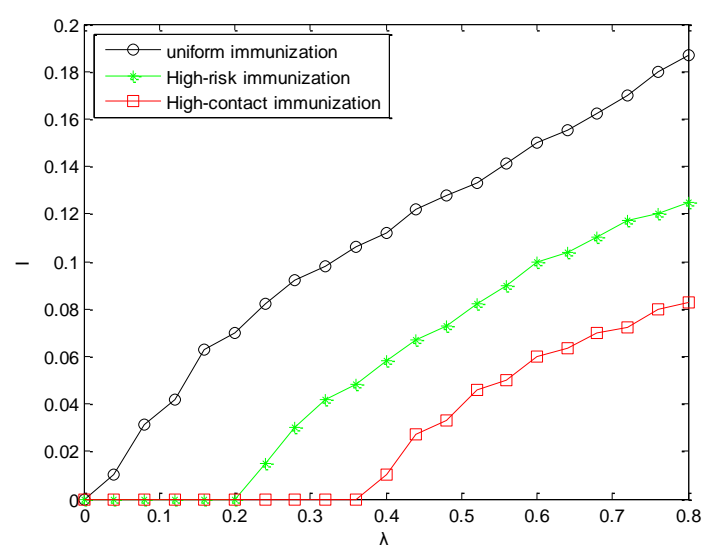

Figure 4. Three different immunization strategies in the BA network $(\mathrm{N}=2000,\langle\mathrm{k}\rangle=4, \delta=0.3$ Infection density varies with propagation probability)

Fig. 4 is the compare of three different immunization strategies in the BA network $(N=2000,<$ $k>=4, \delta=0.3$ ). From the Fig.4 we can see that the effect of uniform immunization is worst among three strategies. High-risk immunization is better than uniform immunization, and the high-contact-first immunization is best. For high-contact-first immunization, the probability that nodes get vaccinated is not all the same. It is varied according to the contact frequency between nodes. And the high-contact-first immunization is a dynamic immunization strategy, and it can eliminate the potential spreading risks in the network.

Fig.5. is the comparison of high-contact-first immunization with different average degree $(N=2000)$. From Fig.5, we can know: when $\langle k\rangle$ gets bigger, the effect gets worse, which is consistent with above conclusions. In fact, when people communicate more frequently, the spread of disease gets faster.

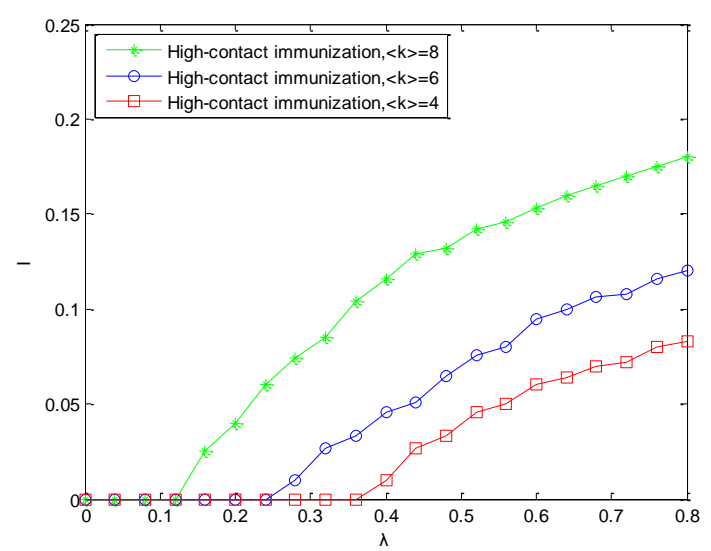

Figure 5. The comparison of high-contact-first immunization with different average degree $(\mathrm{N}=2000$ Infection density varies with propagation probability) 


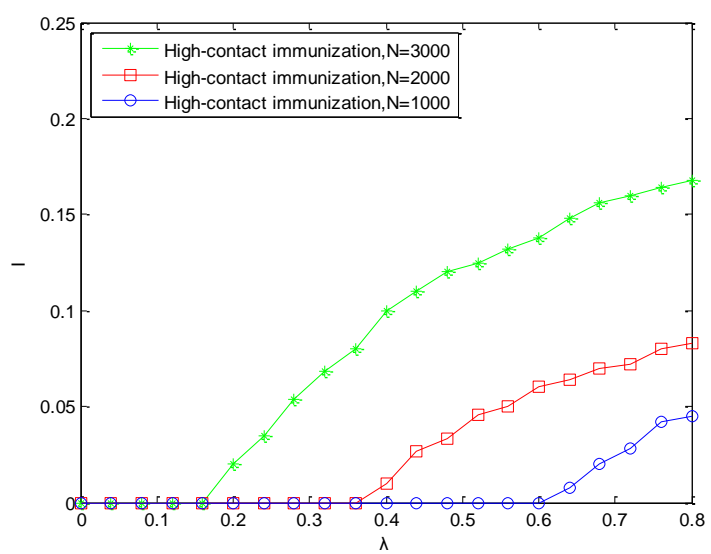

Figure 6. The contrast of effect of high-contact-first immunization on different networks with different scale $\mathrm{N}(<\mathrm{k}>=4$, Infection density varies with propagation probability)

Fig. 6 is the contrast of effect of high-contact-first immunization on different networks with different scale $N$. It indicates propagation probability $\lambda_{c}$ decreases with $N$ increasing. It means that the more population, the more easilyoutbreak infectious diseases, the threshold higher. Whlie the effect gets better when $N$ is smaller, which is also consistent with above conclusions and the situation in real life.

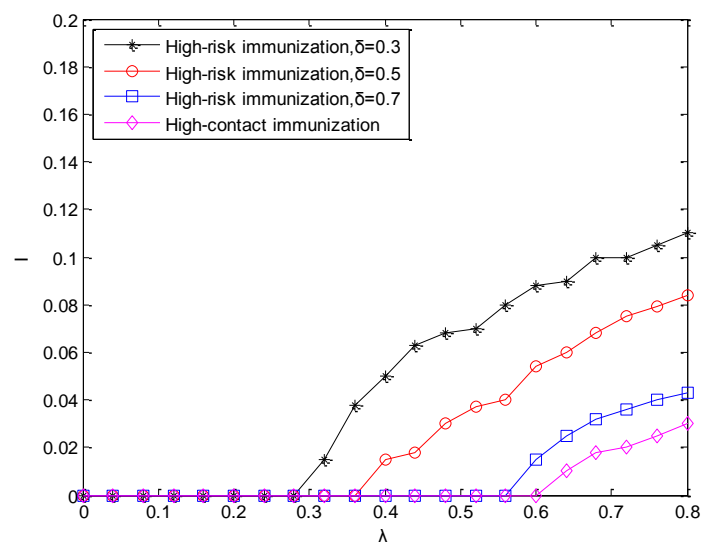

Figure 7. The contrast between high-risk immunization and high-contact-first immunization $(\mathrm{N}=1000,<\mathrm{k}>=4$, the variation of infected density I changes with the function of $\lambda)$

Fig. 7 is the contrast between high-risk immunization and high-contact-first immunization $(N=1000,<k>=4)$, the variation of infected density $I(\lambda)$ changes with the function of $\lambda$.

From Fig.7, it is obvious that the $\delta$ gets bigger, which means more nodes have been vaccinated, the vaccinated effect is better. Compared to high-contact-first immunization, the latter is better. Even $\sigma=0.7$, the effect is still better than high-risk immunization.

And now, how apply the high-contact-first immunization to real life? As we known, the contact of the family members, company colleagues, friends and classmates is more frequently than others. Thus, these population is the first target of immunization when an epidemic occurs. Another problem is how to find an infected individual quickly. Usually, infectious diseases will perform certain external characteristics. So we can use the characteristics to find infected individuals first and vaccinate their family members, company colleagues, friends, classmates and etc. So, the high-contact-first immunization is feasible in real life.

\section{Conclusions}

With the development of human society, the infectious disease cycles of outbreak is getting shorter, and choose an effect immunization strategy becomes more and more important. For a good 
immunization strategy, many factors should be considered in real life, such as politics, economy, culture and region. In this paper, based on the different frequency of interpersonal communication, we designed a dynamic strategy: "high-contact-first immunization strategy", and the results show the high-contact-first immunization is efficient and feasible.

\section{Acknowledgments}

This research is supported by the National Natural Science Foundation of China (No: 61263019), Program for International S\&T Cooperation Projects of Gansu province(No.144WCGA166) and the Doctoral Foundation of LUT.

\section{References}

[1] Chen, Q., Teng, Z., Wang, L. et al.:The existence of codimension-two bifurcation in a discrete SIS epidemic model with standard incidence. Nonlinear Dyn 71 (1-2), 55-73 (2013).

[2] Xia, C., Wang, L., Sun, S. et al.:An SIR model with infection delay and propagation vector in complex networks. Nonlinear Dyn 69 (3), 927-934 (2012).

[3] Chu, X., Zhang, Z., Guan, J. et al.:Epidemic spreading with nonlinear infectivity in weighted scale-free networks. Physica A: Statistical Mechanics and its Applications 390 (3), 471-481 (2011).

[4] Merler, S. and Ajelli, M.:Human mobility and population heterogeneity in the spread of an epidemic. Procedia Computer Science 1 (1), 2237-2244 (2010).

[5] Li, A., Gu, L., and Xu, K. A.:Fast Anomaly Detection for Large Data Centers. Glob Telecomm Conf (2010).

[6] González-Parra, G., Acedo, L., Villanueva Micó, R.-J. et al.:Modeling the social obesity epidemic with stochastic networks. Physica A: Statistical Mechanics and its Applications 389 (17), 3692-3701 (2010).

[7] Wang, L., Teng, Z., and Zhang, L., presented at the Abstract and Applied Analysis, 2014 (unpublished).

[8] Sun, Y., Liu, C., Zhang, C.-X. et al.:Epidemic Spreading on Weighted Complex Networks. Physics Letters A (2014).

[9] Nian, F. and Liu, T., presented at the Abstract and Applied Analysis, 2014 (unpublished).

[10]Lin, Y., Jiang, D., and Wang, S.:Stationary distribution of a stochastic SIS epidemic model with vaccination. Physica A: Statistical Mechanics and its Applications 394, 187-197 (2014).

[11] Li, C.-H., Tsai, C.-C., and Yang, S.-Y.:Analysis of epidemic spreading of an SIRS model in complex heterogeneous networks. Communications in Nonlinear Science and Numerical Simulation 19 (4), 1042-1054 (2014).

[12]Zaidi, F.:Small world networks and clustered small world networks with random connectivity. Social Network Analysis and Mining, 1-13 (2013).

[13] May, R. M. and Lloyd, A. L.:Infection dynamics on scale-free networks. Phys Rev E 64 (6), $066112(2001)$.

[14] Barthélemy, M., Barrat, A., Pastor-Satorras, R. et al.:Velocity and hierarchical spread of epidemic outbreaks in scale-free networks. Physical Review Letters 92 (17), 178701 (2004).

[15] Pastor-Satorras, R. and Vespignani, A.:Epidemic spreading in scale-free networks. Physical review letters 86 (14), 3200 (2001). 
[16] Nian, F. and Liu, T.:High-Risk Immunization Strategies for Multiethnic Regions. Abstract and Applied Analysis 2014, 6 (2014).

[17] Yang, H. X., Wang, W. X., Lai, Y. C. et al.:Control of epidemic spreading on complex networks by local traffic dynamics. Phys Rev E 84 (4) (2011).

[18]Liu, J. and Zhang, T.:Epidemic spreading of an SEIRS model in scale-free networks. Communications in Nonlinear Science and Numerical Simulation 16 (8), 3375-3384 (2011).

[19] Nian, F. and Wang, X.:Efficient immunization strategies on complex networks. Journal of Theoretical Biology 264 (1), 77-83 (2010).

[20] Anderson, R. and May, R., Infectious Diseases in Humans. (Oxford University Press, Oxford, 1992).

[21] Pastor-Satorras, R. and Vespignani, A.:Immunization of complex networks. Phys Rev E 65 (3), 036104 (2002).

[22] Cohen, R., Havlin, S., and Ben-Avraham, D.:Efficient immunization strategies for computer networks and populations. Physical Review Letters 91 (24), 247901 (2003).

[23] Watts, D. J. and Strogatz, S. H.:Collective dynamics of 'small-world'networks. nature 393 (6684), 440-442 (1998).

[24] Pastor - Satorras, R. and Vespignani, A.:Epidemics and immunization in scale - free networks. Handbook of graphs and networks: from the genome to the internet, 111-130 (2005).

[25] Barabási, A.-L. and Albert, R.:Emergence of scaling in random networks. science 286 (5439), 509-512 (1999).

[26] Pastor-Satorras, R. and Vespignani, A.:Epidemic dynamics and endemic states in complex networks. Phys Rev E 63 (6), 066117 (2001). 